\title{
Complicated Maternal Death: Puerperal Sepsis in a Patient with Subclinical Chronic Disease
}

\author{
Samadhi H Dandeniya Arachchi*, Rohan P Ruwanpura \\ Office of the Judicial Medical Officer, Teaching Hospital, Karapitiya, Galle, Sri Lanka.
}

\begin{abstract}
Maternal death is a preventable tragedy. However, in some instances, a combination of different entities might complicate the case and mislead the diagnosis. This is a case of puerperal sepsis during the early postpartum period in a previously healthy primipara due to a chronic disease that has exacerbated. The postmortem examination revealed mildly infected foci of uterine mucosa, massive hepatosplenomegaly and multiple yellow and pale patches in ventricles confirmed microscopically as myocardial infractions)with patent coronaries. Cause of death was determined as puerperal sepsis complicated with chronic anaemia. Culture-negative septicemia has been reported in chronic disease such as malignancies (eg. lymphoma, paraneoplastic syndrome) etc. Generalized sepsis may rapidly arise from miniature foci of sepsis with undiagnosed predisposing conditions. A higher degree of clinical vigilance combines with necessary investigations are essential to establish the proper diagnosis and prompt management. Thorough antenatal screening of pregnant mothers is vital irrespective of their clinical state to avoid preventable maternal deaths.
\end{abstract}

Keywords: Chronic anaemia, maternal death, puerperal sepsis

Received:12 June 2019, Revised version accepted: 23June 2019, Published: 30 June 2018. *Corresponding author: Samadhi DAH, $\triangle$ Email:dahsamadhid@gmail.com, (D) https://orcid.org/0000-0003-4814-6255

Cite this article as: Samadhi DAH, Ruwanpura PR. Complicated Maternal Death: Puerperal Sepsis in a Patient with Subclinical Chronic Disease. Medico-Legal Journal of Sri Lanka, 2019;7(1):22-5. DOI: http://doi.org/10.4038/mljsl.v7i1.7390

Copyright: @ 2019 with the Medico-legal Journal of Sri Lanka.

This is an open-access article distributed under the terms of the Creative Commons Attribution 4.0 International License, which permits unrestricted use, distribution and reproduction in any medium provided the original author and source are credited.

\section{Introduction}

Maternal death is defined by World Health Organization (WHO) as "The death of a woman while pregnant or within 42 days of termination of pregnancy, irrespective of the site and duration of the pregnancy from any cause related to or aggravated by the pregnancy or its management, but not from accidental or incidental causes". ${ }^{[1]}$ It is identified as a preventable tragedy worldwide. ${ }^{[2]}$ Majority of these deaths are due to direct causes and postpartum haemorrhage, eclampsia and sepsis are at the top of the list. ${ }^{[3]}$ Heart disease and anaemia are the main contributors of indirect maternal deaths. ${ }^{[3,4]}$ However, in some instances, a combination of several entities might complicate the clinical picture resulting in misdiagnosis.

\section{Case report}

A29-year-old primi mother who was was apparently well before and during pregnancy, and her antenatal haemoglobin level has been maintained around $9.5 \mathrm{mg} / \mathrm{dl}$ throughout the pregnancy. She developed signs of sepsis during the early postpartum period following a symptomless interval of two weeks. She underwent an emergency caesarean section due to obstructed labour, delivered a healthy infant and was discharged from the hospital on the fourth day. She was readmitted after two weeks with fever and abdominal pain, and the haemoglobin was $5 \mathrm{mg} / \mathrm{dl}$ with massive hepatosplenomegaly. She died on the second day after admission while being investigated and treated with broad-spectrum antibiotics together with repeated blood transfusions.

The postmortem examination revealed mildly infected foci of uterine mucosa (Figure 1), massive hepatosplenomegaly (weight of the liver was $4315 \mathrm{~g}$, and the spleen was 495g) (Figure 2) and multiple patchy myocardial infarctions (Figure 3) in the left ventricular wall with apparently patent coronaries. No genital injuries were noted. 


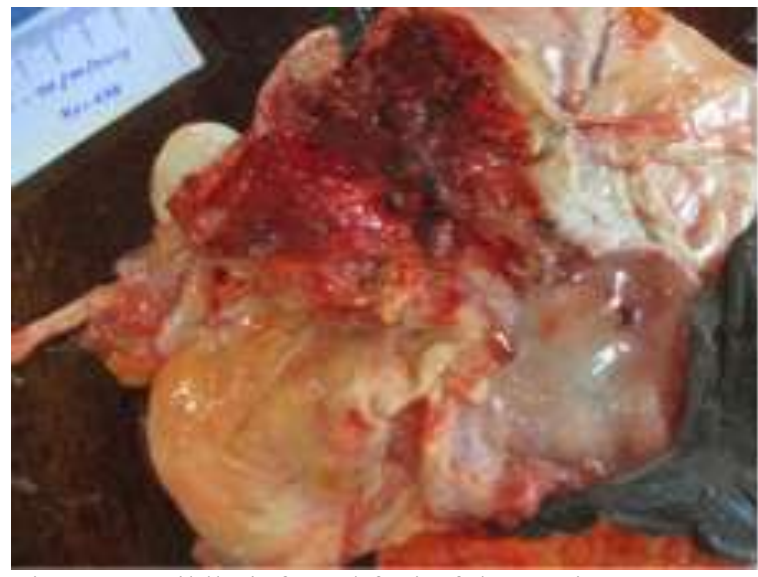

Figure 1. Mildly infected foci of the uterine mucosa

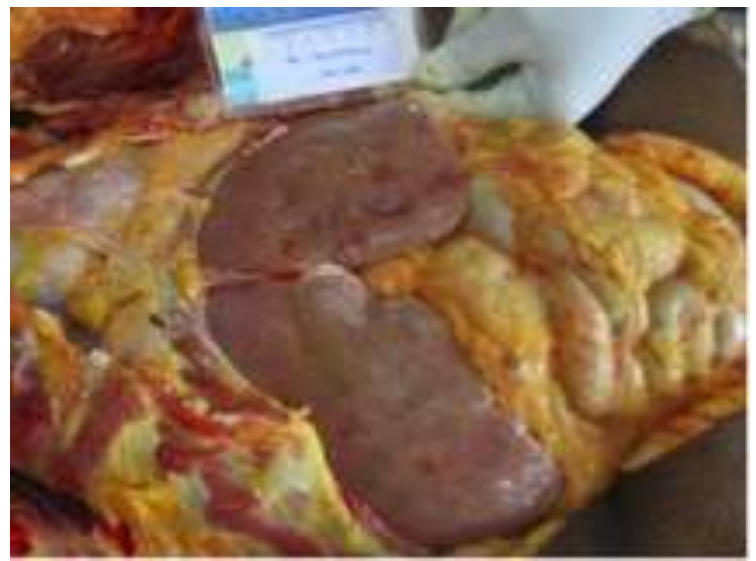

Figure 2. Massive hepatosplenomegaly

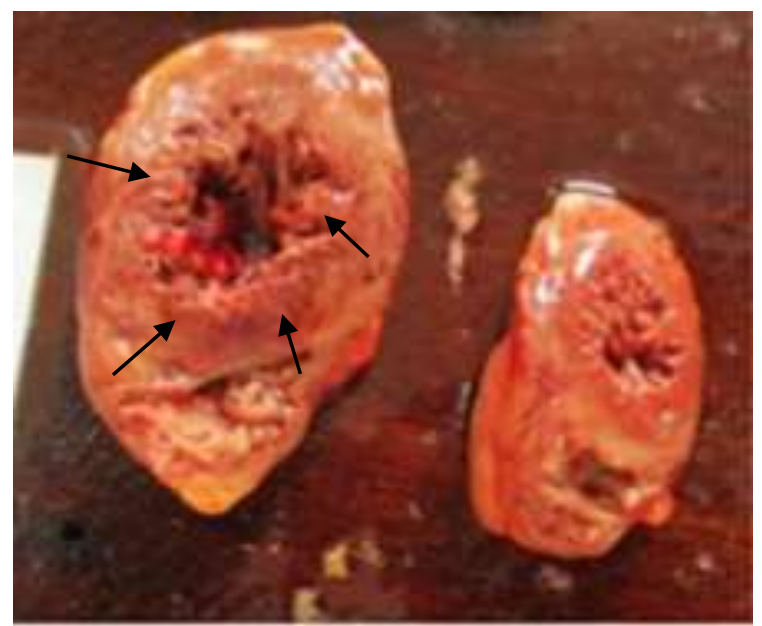

Figure 3. Multiple patchy myocardial infarctions (Black arrows)

The histopathological investigations showed multiple fibrin thrombi in branches of coronary arteries (Figure $4 \& 5$ ) and pulmonary and hepatic vasculature, reactive splenomegaly, and fibro-fatty changes with cell necrosis in the liver (Figure6). Lung tissues showed features suggestive of diffuse alveolar damage (Figure
7). Renal tubular necrosis is identified with the same vascular lesions, as mentioned above. Bone marrow biopsy suggestive (but inconclusive due to autolytic changes) of monomorphic lymphoblastic infiltration (Figure 8).

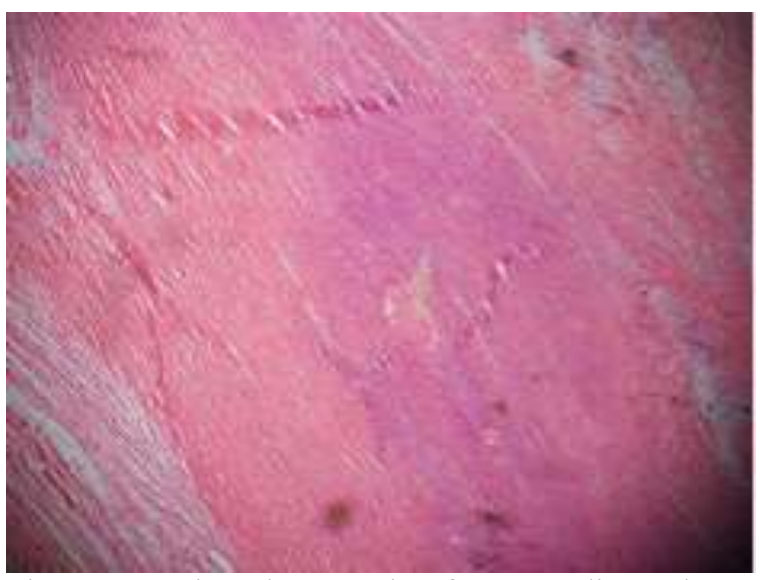

Figure 4. Microphotograph of myocardium shows multiple fibrin thrombi in branches of coronary arteries (H\&E x 40)

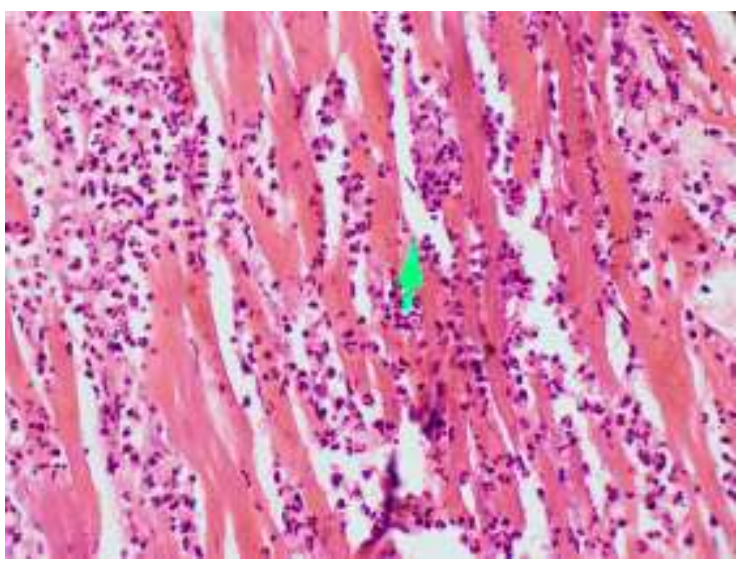

Figure 5. Microphotograph of the heart shows massive necrosis of myofibrils surrounded by predominantly neutrophilic mixed cellular infiltration (H\&E x 100)

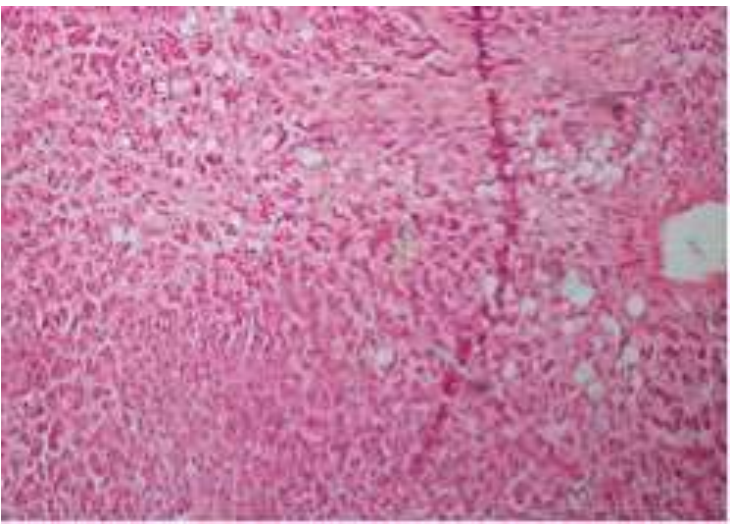

Figure 6. Microphotograph of the liver shows fibro- 
fatty changes with cell necrosis in the liver (H\&E x 100)

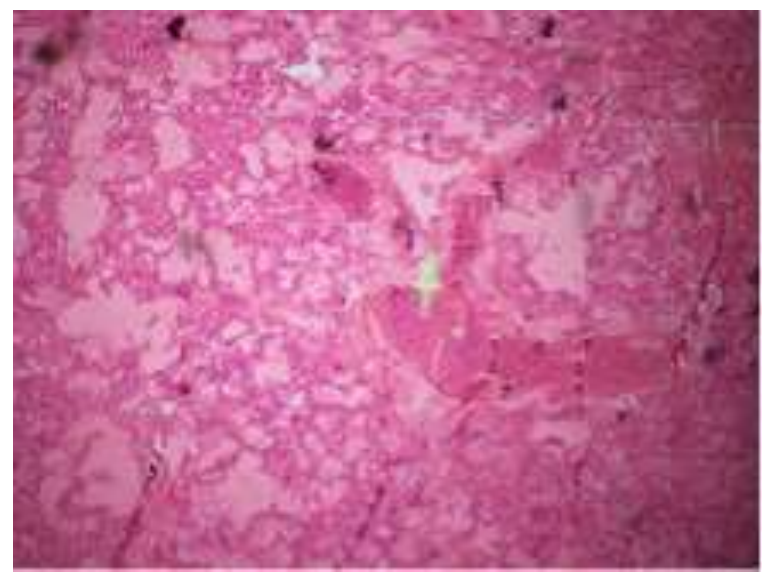

Figure 7. Microphotograph of lung shows diffuse alveolar damage (H\&E x 40)

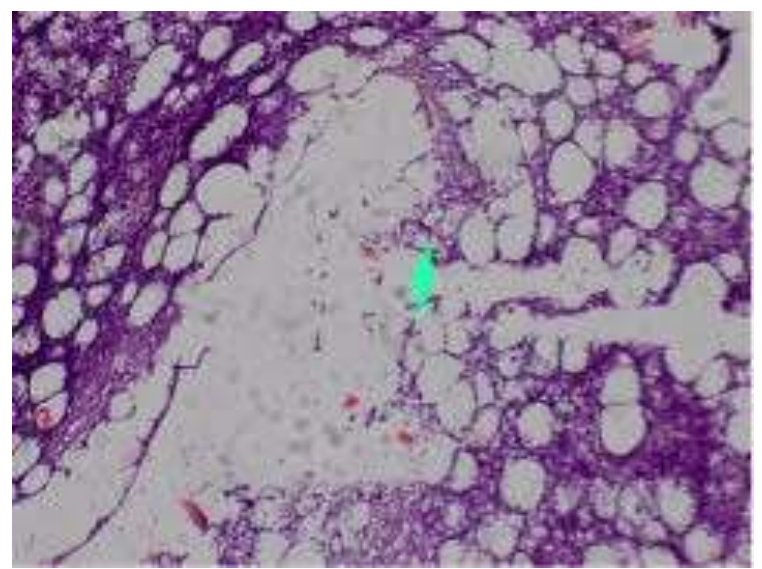

Figure 8. Microphotograph of bone marrow shows monomorphic lymphoblastic infiltration (H\&E x 100)

Antemortem and postmortem cultures were negative. The cause of death was determined as puerperal sepsis complicated with anaemia complicating a chronic disease.

\section{Discussion}

Maternal mortality is a reliable indicator of the quality of the health care system and the socio-economic status of any country. According to WHO most of the maternal deaths are reported in developing countries, and it is proven that the majority of them could have been prevented with timely screening and prompt management. ${ }^{[5]}$

The victim of this case was apparently well before and during pregnancy, and antenatal haemoglobin level has been maintained within normal range throughout the pregnancy. In fact, mild reduction of haemoglobin level had not been considered abnormal during routine follow up as it is a natural regulatory mechanism due to haemo-dilution in pregnancy, and also the mother was symptomless throughout. It is a known reported fact that the physiological anaemia in pregnancy could be aggravated by various communicable and noncommunicable diseases such as Malaria, HIV, Malignancy etc. ${ }^{[6]}$ Presence of massive hepatosplenomegaly and bone marrow abnormalities in the present case are suggestive of a chronic underlying condition that appeared to be sub-clinical and not detected during pregnancy. Diagnosis of hepatosplenomegaly is somewhat challenging during pregnancy even with ultrasound imaging in the absence of any related symptom or illness. Hence, under these circumstances, the misdiagnosis of the chronic medical condition during her antenatal visits cannot be attributed to medical negligence.

The size and weight of the liver of this patient were almost three times of normal at the time of death. All the other causes for massive hepatosplenomegaly like malaria, hepatitis, HIV etc. have been excluded during ward stay and it has been provisionally diagnosed as a case of autoimmune haemolytic anaemia. The patient has succumbed to her illness before concluding a diagnosis with further bone marrow studies. The postmortem bone marrow biopsy suggestive of monomorphic cellular infiltration, most probably lymphoblastic in origin, but the postmortem autolytic changes made the diagnosis inconclusive. The histology and immune histochemistry studies have limited values in postmortem bone marrow samples. [7,8] However, lymphomas are well-reported malignancies, which cause anaemia in pregnancy. ${ }^{[6]}$ Absence of lymphadenopathy is not exclusive of lymphoma in this case, as all the other clinicopathological findings are in favour of the diagnosis.

Interestingly, all the blood, urine and tissue cultures were negative in antemortem and postmortem samples. However, the culture negative septicaemia has also been reported in chronic diseases such as malignancies (eg. lymphoma, paraneoplastic syndrome), etc. ${ }^{[9]}$ The victim showed septic features and other biochemical markers in keeping with the severe inflammatory response syndrome (SIRS). Furthermore, the presence of micro-thrombi in vasculature together with the antenatal haematological findings confirms the development of diffuse intravascular coagulation, a common terminal outcome of generalized sepsis. The diffuse thrombosis of the vasculature of the myocardium following DIC could have lead to multiple pale and yellowish patchy areas suggestive of myocardial necrosis though coronaries remained patent. It has further been 
confirmed by histology and differentiated from neutrophilic myocarditis due to the presence of massive myocardial cell necrosis surrounded by mixed cellular reaction pathognomonic of myocardial infarction. According to other studies, generalized sepsis may rapidly arise from miniature foci of sepsis with undiagnosed predisposing conditions. ${ }^{[10]}$

In turn, increased stress associated with chronic illness may alter perinatal outcomes. Pregnant women with chronic disease may benefit from interventions aimed at helping them balance the blessings and burdens associated with the symptoms of pregnancy. ${ }^{[1]}$

\section{Conclusion}

This case emphasizes the importance of thorough antenatal screening of pregnant mothers irrespective of their clinical state to avoid preventable maternal deaths. Severe sepsis, which brings fatal outcome from mild infective foci is reported in expectant mothers with subclinical chronic disease such as haematological malignancies as seen in this case. A higher degree of clinical vigilance is essential in such situations to overcome severe complications and fatal outcome.

\section{Disclosure statement}

Conflicts of interests: The authors declare that they have no conflicts of interests.

Funding: None

\section{References}

1. Khan KS, Wojdyla D, Say L, Gülmezoglu AM, Van Look PF. WHO analysis of causes of maternal death: a systematic review. The lancet. 2006 Apr 1;367(9516):1066-74.PMID: 16581405

2. Begum S, Begum I. Analysis of maternal mortality in a tertiary care hospital to determine causes and preventable factors. Journal of Ayub Medical College Abbottabad. 2003;15(2): 49-4. PMID: 14552250

3. Jafarey SN. Maternal mortality in Pakistancompilation of available data. J Pak Med Assoc. 2002 Dec;52(12):539-44. PMID: 12627900

4. Igwegbe AO, Eleje GU, Ugboaja JO, Ofiaeli RO. Improving maternal mortality at a university teaching hospital in Nnewi, Nigeria. International Journal of Gynecology\& Obstetrics. 2012 Mar;116(3):197-200.PMID: 22196989

5. World Health Organization. Maternal mortality: to improve maternal health, barriers that limit access to quality maternal health services must be identified and addressed at all levels of the health system: fact sheet. World Health Organization; 2014. Available at https://www.who.int/newsroom/fact-sheets/detail/maternal-mortality. Date accessed 10 May 2019

6. Gangopadhyay R, Karoshi M, Keith L. Anemia and pregnancy: a link to maternal chronic diseases. International Journal of Gynecology\& Obstetrics. $2011 \quad$ Nov;115:S11-5.PMID: 22099433

7. Burkhardt R, Kettner G, Böhm W, Schmidmeier M, Schlag R, Frisch B, Mallmann B, Eisenmenger W, Gilg TH. Changes in trabecular bone, hematopoiesis and bone marrow vessels in aplastic anaemia, primary osteoporosis, and old age: a comparative histomorphometric study. Bone. 1987 Jan 1;8(3):157-64.PMID:3606907

8. Webb DI, Ubogy G, Silver RT. Importance of bone marrow biopsy in the clinical staging of Hodgkin's disease. Cancer. 1970 Aug;26(2):3137.PMID: 5451214

9. Mancini N, Clerici D, Diotti R, Perotti M, Ghidoli N, De Marco D, Pizzorno B, Emrich T, Burioni R, Ciceri F, Clementi M. Molecular diagnosis of sepsis in neutropenic patients with haematological malignancies. Journal of medical microbiology. 2008 May 1;57(5):601-4.PMID: 18436593

10. Balk RA. Severe sepsis and septic shock: definitions, epidemiology, and clinical manifestations. Critical care clinics. 2000 Apr 1;16(2):179-92.PMID:10768078

11. Tyer-Viola LA, Lopez RP. Pregnancy with chronic illness. Journal of obstetrics, gynaecology and neonatal nursing. 2014;43(1):25-37.PMID: 24428144 